\title{
Soft X-Ray Microscopy; A New Technology for Examination of Parasitic Specimens
}

\author{
W. J. Kozek, ${ }^{*}$ A. Nair, ${ }^{* *}$ G. Denbeaux, ${ }^{* *}$ C. Larabell, ${ }^{* * *}$ J. Brown, ${ }^{* *}$ C. R. Sterling, ${ }^{* * * *}$ \\ S. Garlapati, ${ }^{* * * * *}$ C. C. Wang***** and W. Meyer-Ilse** \\ *Department of Microbiology and Medical Zoology, Medical Sciences Campus, University of \\ Puerto Rico, San Juan, PR, 00936-5067 \\ ** Center for X-Ray Optics, Advanced Light Source and ***Division of Life Sciences, E. O. L. \\ Berkeley National Laboratory, Berkeley, CA, 94720 \\ **** Department of Veterinary Sciences, School of Veterinary Medicine, University of Arizona, \\ Tucson, AZ, 85721 \\ *****Department of Chemistry, School of Pharmacy, University of California, San Francisco, CA, \\ 94143-0446
}

Construction of the XM-1 microscope, located on beamline 6.1.2 of the Advanced Light Source Facility at the E. O. L Berkeley National Laboratory, has enabled the application of soft X-ray microscopy for examination of biological specimens by providing a high thrughput of high-spatial resolution images from hydrated samples up to $10 \mu \mathrm{m}$ thick. Previous biological applications of soft X-ray microscopy included examination of a variety of cells and unicellular organisms, including intracellular malaria parasites $[1,2,3]$ and tracing intracellular distribution of proteins and nucleic acids[4]. To determine whether this technique could have wider application in life sciences and allow morphological studies of multicellular organisms and fragile protozoa, we have examined by soft X-ray microscopy glutaraldehyde-fixed newborn larvae of Trichinella spiralis, microfilariae of Dirofilaria immitis, trophozoites of Giardia lamblia and oocysts of Cryptosporidium parvum. Radiation damage to the specimens was minimal. In the vacuolated cytoplasm of G. lamblia trophozoite, the following internal structures are readily discernible: the two nuclei, each with its nucleolus, median bodies, located almost at right angle to the axostyle that originates in the central area anterior to the nuclei, and the anterior and posterior flagella (Fig. 1). Examination of multicellular organisms revealed aspects of their internal organization and arrangement of organ precursors that are extremely difficult to obtain by other microscopy techniques. New information obtained about the structure of $T$. spiralis larvae (Fig. 2) indicated that the larva has attained a remarkable degree of development and differentiation that was hitherto not suspected; the relationship between the cellular components, including the Excretory and the R1-Anal Vesicle complexes, was elucidated in the microfilaria; and the development of sporozoites within the oocysts of $C$. parvum (Figs. 3,4) could be discerned. These studies have demonstrated that the unique capabilities of soft X-ray microscopy can be successfully applied to examine the structure of protozoa and small, multicellular organisms. Soft X-ray microscopy can provide new information unattainable by other microscopy techniques, and it is also especially useful to correlate and integrate results obtained by scanning and transmission electron microscopy. 


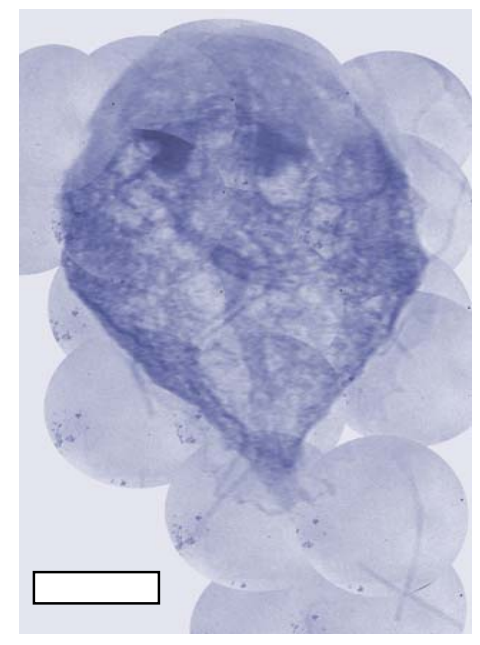

FIG. 1 Soft X-ray montage of G. lamblia trophozoite. Bar $=10 \mu \mathrm{m}$.

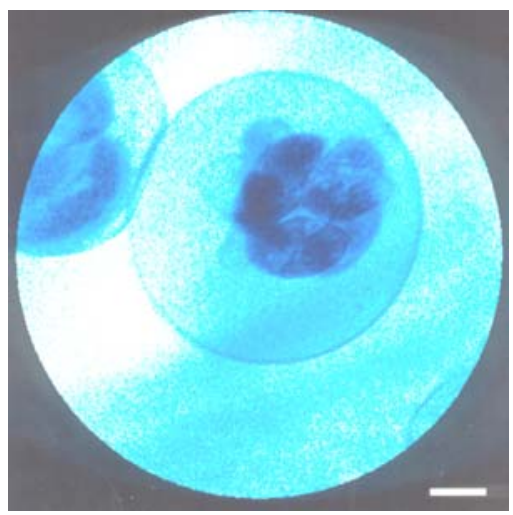

FIG. 3. Dividing sporozoites in C. parvum oocyst. $\mathrm{Bar}=1 \mu \mathrm{m}$.

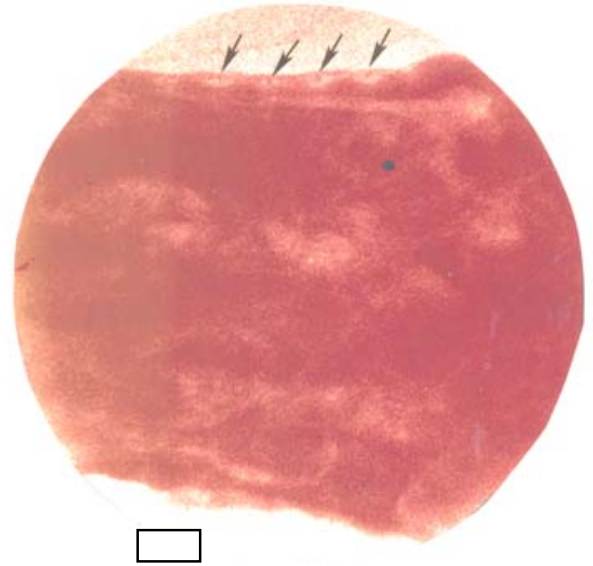

FIG. 2. Newborn larva of T. spiralis. Arrows indicate openings of bacillary bands. Bar $=1 \mu \mathrm{m}$.

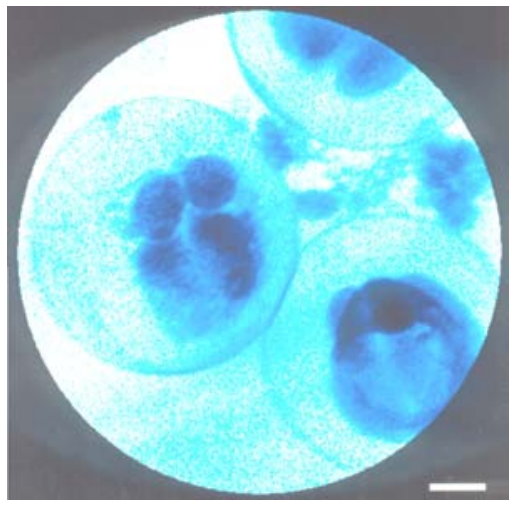

FIG. 4. Four sporozoitess in

C. parvum oocyst. $\mathrm{Bar}=1 \mu \mathrm{m}$.

\section{REFERENCES}

[1] W. Meyer-Ilse et al., Synch. Rad. News 8 (1995) 29.

[2] C. Magowan et al., Proc. Natl. Acad. Sci. USA 94 (1997) 6222.

[3] J. Yeung et al., (1998) Mol. Pathol. Pharmacol. 99 (1998) 245.

[4] C. A. Larabell et al., X-Ray Microscopy: Proc 6th Int. Conf.(2000) 107.

[5] Supported, in part, by an appointment to the Research Participation Program at the E. O. L. Berkeley National Laboratory administered by the Oak Ridge Institute for Sciences and Education through an interagency agreement between the U.S. Department of Energy and the LBNL; the Director, Office of Energy Research, Office of Basic Energy Sciences, Material Sciences Division of the U.S. Department of Energy, under a contract No. DE-Aco3-76SF00098, and Cooperative Agreement DOE/EPSCoR De-FCO2-91ER75674, NIH R01 grant Al-30475 and the RCMI Award RR03051 from the NCRR, National Institutes of Health). 